\title{
Preparación de un Adhesivo Sensible a la Presión (PSA) con la Incorporación de Nanopartículas de ZnO. Estudio de sus Propiedades Fisicoquímicas y Antimicrobianas
}

\section{Preparation of a Pressure Sensitive Adhesive (PSA) with the ZnO Nanoparticles Incorporation. Study of its Physicochemical and Antimicrobial Properties}

\author{
S. N. Ramírez-Barrón', S. Sánchez-Valdés' , B. A. Puente-Urbina', S. Martínez-Montemayor'1, S. C. Esparza-González², \\ R. Betancourt-Galindo' \\ 'Centro de Investigación de Química Aplicada, CIQA \\ ${ }^{2}$ Facultad de Medicina de Saltillo, UAdeC
}

\section{RESUMEN}

Se describe el proceso para obtener un adhesivo sensible a la presión (PSA). Este PSA está formado por un copolímero de acrilato de 2-etilhexil (2-EHA) / metacrilato de metilo (MMA) en una relación 80:20 que se polimerizó mediante una técnica de polimerización en emulsión. Se añadieron nanopartículas de óxido de zinc (NPZnO) a este copolímero, que se sintetizaron previamente y se modificaron superficialmente con 3-aminopropil-3-toxisilano (APTES) y dimetilsulfóxido (DMSO) para mejorar su dispersión en la matriz de copolímero. Los nanocompuestos obtenidos se caracterizaron por espectroscopía infrarroja (FTIR), calorimetría diferencial de barrido (DSC) y pruebas de adhesión al delaminado. Además, se determinó la actividad antimicrobiana contra $S$. aureus y $S$. pyogenes, así como la citotoxicidad en células humanas (HeLa). Los resultados demostraron que la adición de las nanopartículas de NPZnO al copolímero incrementa la temperatura de transición vítrea (Tg) así como las propiedades antimicrobianas del adhesivo mejorando a su vez su adhesión superficial. Con respecto al comportamiento adhesivo, el PSA con NPZnO sin modificar mostró una mayor resistencia al delaminado, esto quiere decir que las nanopartículas incrementan la fuerza cohesiva y proporcionan resistencia a temperaturas elevadas, lo cual sería beneficioso a su aplicación final. Finalmente, los resultados de citotoxicidad mostraron que la incorporación de NPZnO al PSA disminuye la viabilidad celular, sin embargo no se considera tóxico acorde a la norma ISO 10993 test for in vitro cytotoxicity.

Palabras Clave: Nanopartículas de ZnO; modificación de la superficie; polimerización en emulsión; propiedades antimicrobianas; citotoxicidad 


\section{ABSTRACT}

The process for obtaining a pressure sensitive adhesive (PSA) is described. This PSA is formed by an acrylate copolymer of 2-ethylhexyl (2-EHA) / methyl methacrylate (MMA) in an 80:20 ratio which was polymerized by emulsion polymerization technique. Zinc oxide nanoparticles (NPZnO) were added to this copolymer, which were previously synthesized, and surface modified with 3-aminopropyltretoxysilane (APTES) and dimethyl sulfoxide (DMSO) to improve its dispersion in the copolymer matrix. The obtained nanocomposites were characterized by infrared spectroscopy (FTIR), differential scanning calorimetry (DSC) and T-peel adhesion tests. In addition, the antimicrobial activity against $S$. aureus and $S$. pyogenes as well as the cytotoxicity in human cells (HeLa) were determined. The results demonstrated that the $\mathrm{ZnO}$ nanoparticles incorporation enhanced the glass transition temperature (Tg) and the antimicrobial activity of PSA copolymer as well as its surface adhesion. It was confirmed that NPZnO modification with APTES increased its antimicrobial activity. Regarding adhesive behavior, PSA with unmodified NPZnO showed a greater peel resistance. This indicates that these nanoparticles enhances the cohesive force and induces a better high temperature performance, which is beneficial for the final application. Finally, cytotoxicity results showed that the incorporation of NPZnO to PSA decreases the cell viability, however this PSA is not toxic according to the standard ISO 10993 test for in vitro cytotoxicity.

KEYWORDS: ZnO nanoparticles; surface modification; emulsion polymerization; antimicrobial properties; cytotoxicity

\section{Correspondencia}

DESTINATARIO: Rebeca Betancourt Galindo INSTITUCIÓN: Centro de Investigación de Química

Aplicada, CIOA

DIRECCIÓN: Blvd. Enrique Reyna Hermosillo \#140, C.P. 25294, Saltillo, Coahuila, México CORREOELECTRÓNICO: rebeca.betancourt@ciqa.edu.mx

\section{Fecha de recepción:}

18 de septiembre de 2018

Fecha de aceptación:

10 de enero de 2018 


\section{INTRODUCCIÓN}

Los adhesivos sensibles a la presión (PSA) son materiales viscoelásticos que, en su estado seco y a temperatura ambiente, se vuelven pegajosos cuando se aplica una pequeña presión. Debido a estas propiedades, los PSA tienen una gran cantidad de aplicaciones, desde la industria de la construcción hasta la medicina. Los PSA se han sintetizado mediante polimerización en emulsión y se han incorporado una amplia variedad de rellenos nanométricos para modificar sus propiedades o incluso añadir nuevas características. Las nanopartículas de $\mathrm{ZnO}$ (NPZnO) son materiales inorgánicos que se pueden incorporar en una matriz polimérica. Las propiedades intrínsecas del PSA dependen de la fase del polímero y de las propiedades antimicrobianas y citotóxicas del material inorgánico de relleno. ZnO tiene una amplia gama de aplicaciones en ingeniería, medicina y aeronáutica, entre otros. Dicha aplicación incluye dispositivos automotrices, sistemas de comunicación, sistemas biológicos tales como biomateriales para ingeniería de tejidos, polímeros de memoria de forma como interruptores moleculares, biosensores, diagnóstico de laboratorio y liberación de fármacos ${ }^{[1]}$. Existen diferentes factores que afectan la funcionalidad del PSA, entre los que se encuentran la composición, la morfología de las partículas, la resistencia adhesiva, la flexibilidad, entre otros ${ }^{[2]}$. Se han publicado varios trabajos relacionados con la síntesis de PSA con diferentes partículas nanoestructuradas, para mejorar las propiedades mecánicas y de liberación del fármaco, o aumentar la conductividad eléctrica. Por lo tanto, Jingyeong O. (2009) ${ }^{[3]}$ prepararon composiciones de nanoarcillas de montmorillonita de poliacrilato (MMT) en diversos contenidos de arcilla por mezcla mecánica y polimerización en emulsión in situ. Descubrieron que la temperatura de transición vítrea ( $\mathrm{Tg}$ ) del polímero y las propiedades viscoelásticas dependen de la concentración final de MMT. Las propiedades mecánicas de PSA usando EA/EHA llena de NaMMT fueron estudiadas

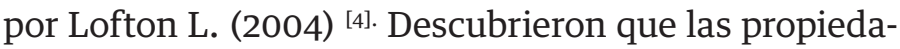
des adhesivas se ven fuertemente afectadas por el tipo y la cantidad de nanoarcillas incorporadas. Por ejemplo, la fuerza de delaminado y adherencia disminuyó gradualmente con una mayor cantidad de nanoarcilla de MMT. Y se observó un aumento sustancial en el esfuerzo cortante. El contenido máximo determinado de arcilla modificada fue $1 \%$ en peso. Mientras que se observó una influencia moderada sobre la adherencia, la resistencia al despegue y el esfuerzo cortante cuando se incorporaron nanoarcillas no modificadas ${ }^{[5,6]}$.

Otra investigación presenta la incorporación de silicato de sodio a nanoescala a Poli (2-EHA-co-AA) con el fin de mejorar la absorción de sodio-cloxacilina. También Wang et al. (2006) [7], desarrollaron un adhesivo de polibutilacrilato y SWNT (nanotubos de carbono de pared simple) para aumentar la conductividad eléctrica. Los SWCNT se funcionalizaron con poli (alcohol vinílico) (PVA), en una proporción de 0,3\% en peso. Los resultados demostraron que la conductividad aumenta en diez órdenes de magnitud. También en este umbral, la adhesión cambia y se observó un aumento en la pegajosidad. De la misma manera, la energía de adhesión aumenta aproximadamente un $85 \%$ con la adición de PVA-SWNT a la misma concentración. Aunque hay varios informes sobre la preparación de nanocompuestos adhesivos de PSA acrílico, no se han encontrado informes que impliquen la incorporación de NPZnO, modificadas o no modificadas en la superficie, en la matriz de polímero adhesivo PSA.

Por lo tanto, en este trabajo se discute la síntesis de NP esféricas de ZnO y su incorporación a PSA. Las NPZnO se modificaron en superficie con dos agentes diferentes: 3-aminopropiltrietoxisilano (APTES) y dimetilsulfóxido (DMSO) a contenidos de 0.1, 0.2 y $0.3 \%$ en peso. Las nanopartículas obtenidas se caracterizaron por difracción de rayos X (XRD) y microscopía electrónica de transmisión (TEM). El compuesto PSA/NP obtenido se estudió mediante microscopía electrónica de transmisión (TEM), resistencia al delaminado y pruebas antimicrobianas contra dos microorganismos diferen- 
tes: Staphylococcus aureus (S. aureus) y Streptococcus pyogenes (S. pyogenes), y finalmente, se realizaron pruebas de citotoxicidad en los adhesivos obtenidos.

\section{METODOLOGÍA}

\section{Materiales}

Acrilato de 2 etilhexilo (2-EHA), Metilmetacrilato (MMA), ácido acrílico (AA), persulfato de amonio (APS), Latemul-180, dodecanotiol, hidroquinona (HQ) acetato de zinc $\left(\mathrm{Zn}(\mathrm{Ac})_{2} \cdot 2 \mathrm{H}_{2} \mathrm{O}\right)$, hidróxido de sodio $(\mathrm{NaOH})$, dimetilsulfoxido (DMSO) y aminopropiltrimetoxisilano (APTES) todos con 98\% de pureza y de Sigma Aldrich. Etanol fue obtenido de J.T. Baker y agua desionizada de un sistema de columnas de intercambio iónico (Cole-Parmer Instruments).

\section{Preparación de las nanopartículas de ZnO (NPZnO)}

NPZnO fueron sintetizadas preparando una solución etanólica de $0.06 \mathrm{M}$ de $\mathrm{Zn}(\mathrm{Ac})_{2} \cdot 2 \mathrm{H}_{2} \mathrm{O}$ que fueron colocadas en un reactor. Enseguida la mezcla fue puesta en reflujo a $80^{\circ} \mathrm{C}$ por $2 \mathrm{~h}$ a $100 \mathrm{rpm}$. Después de agregar una solución aucosa que contenía $0.22 \mathrm{M}$ de $\mathrm{NaOH}$, la mezcla resultante se puso en marcha durante $12 \mathrm{~h}$ a temperatura ambiente y el precipitado se hizo pasar por centrifugado tres veces con el $500 \mathrm{~mL}$ de etanol y se secó en una estufa a $80^{\circ} \mathrm{C}$ durante $24 \mathrm{~h}$.

\section{Modificación superficial}

La modificación superficial de las NPZnO fue llevada a cabo colocando $10 \mathrm{~g}$ de nanopartículas en un matraz bola de 3 bocas provisto de un condensador al que se adicionaron $100 \mathrm{~mL}$ de octano como solvente. Los diferentes agentes modificantes (dimetil sulfóxido y 3 aminopropiltrietoxisilano) se añadieron en una relación en masa de 2:1. La reacción se llevó a cabo mediante reflujo durante $3 \mathrm{~h}$ a $80^{\circ} \mathrm{C}$ y agitación constante. Después el sistema fue enfriado, el material se pulverizó y secó durante $12 \mathrm{~h}$ a $80^{\circ} \mathrm{C}$ para su posterior caracterización.

\section{Sintesis de adhesivo sensible a la presión (PSA)}

Diferentes concentraciones de nanopartículas (0.1, 0.2 y $0.3 \%)$, sin y con modificación superficial con los agentes APTES y DMSO, fueron dispersadas en la mezcla de monómeros 2-EHA/MMA en una relación en masa de 4:1; es decir, se añadieron $16 \mathrm{~mL}$ de 2-EHA y 2 mL de MA del agente de transferencia de cadena AA, así como 2 partes por 100 partes de monómero en peso (phm) utilizando un sonificador Branson W700 con un $38 \%$ de potencia por 15 minutos, siendo esta la fase oléica en el sistema de polimerización. Asimismo, se preparó una solución micelar en una relación peso surfactante/agua (Latemul 180/ $\mathrm{H}^{2} \mathrm{O}$ ) de 0.8/99.2\%, agitando durante 30 minutos; luego se adicionó la fase oléica y se mantuvo por 5 minutos. Después, los componentes de la emulsión se colocaron en un reactor enchaquetado con flujo de nitrógeno para prevenir la oxidación. La reacción se realizó a una velocidad de $400 \mathrm{rpm}$, a $80^{\circ} \mathrm{C}$, durante $2 \mathrm{~h}$. También se controló el pH con una solución de $\mathrm{NaOH}$ al 10\% como agente neutralizante para modificar la acidez de la emulsión.

\section{Caracterización}

Las NPZnO fueron caracterizadas mediante un análisis de XRD llevado a cabo en un difractómetro SIEMENS D-5000 con radiación CuK $\alpha$ para identificar la fase cristalina de las nanopartículas. Las NPZnO sin y con modificación y el PSA fueron caracterizadas con un JEOL 1200XII TEM para obtener la distribución de tamaños de partícula y la incorporación de las nanopartículas en el PSA. El análisis de FT-IR fue llevado a cabo en un espectrofotómetro Nicolet Magna 5500 utilizando ATR de las muestras de los PSA obtenidos para identificar las bandas de absorción presentes en cada uno de los materiales sintetizados. La determinación de la temperatura de transición vítrea (Tg) se determinó mediante calorimetría diferencial de barrido (DSC) en un DSC modelo 2920 (TA Instruments) a presión constante en una amplitud de $\pm 1^{\circ} \mathrm{C}$ cada 60 s a una velocidad de $10^{\circ} \mathrm{C} / \mathrm{min}$ hasta temperatura ambiente. 


\section{Resistencia a la delaminación}

Las pruebas mecánicas que se practicaron a los adhesivos sin y con NPZnO, para determinar la fuerza de delaminación y de corte, se realizaron depositando material adhesivo en una película de polietileno, las cuales fueron puestas a secar a temperatura ambiente por $48 \mathrm{~h}$ para evaporar el agua y el monómero residual presente. Después se cortaron tiras de $25.4 \mathrm{~mm}$ de ancho, preparadas mediante un recubrimiento preciso de $0.03 \mathrm{~mm}$ de espesor del adhesivo con y sin nanopartículas aplicado, bajo condiciones fijadas por los estándares de ASTM, a una temperatura de $23{ }^{\circ} \mathrm{C}$ y $50 \%$ de humedad relativa y bajo los procedimientos establecidos por la ASTM para la evaluación de los materiales sintetizados en este trabajo, Pelaje: D3330/D 3330M-04 y adhesión de corte: D3654/D 3654M-02, la cual marca una velocidad de prueba de $300 \mathrm{~mm} / \mathrm{min}$ (11.8100 in/ min). Se utilizó un rodillo de $2.06 \mathrm{Kg}$ el cual se pasó tres veces por la muestra para ejercer presión sobre ella. Los ensayos se llevaron a cabo en una máquina de ensayos universal marca Instron.

\section{Determinación de la actividad antimicrobiana de las nanopartículas y de los adhesivos}

Con la finalidad de evaluar la sensibilidad antimicrobiana de los nanocompuestos y las nanopartículas en estudio, se procedió a la realización de pruebas microbiológicas, las cuales comprenden el método de difusión con disco en agar de acuerdo con los estándares marcados por CLSI (Instituto de Estándares Clínicos y de Laboratorio). En el caso de las nanopartículas se pusieron discos de nanopartículas de $1 \mathrm{~cm}$ de diámetro obtenidos por compresión y de los nanocompuestos se colocaron muestras de $2 \mathrm{~cm}$ de diámetro provistas de un soporte de polietileno.

La suspensión bacteriana se extendió en 3 planos sobre la superficie de la placa de agar sangre (AS) para S. pyogenes y Mueller Hinton $(\mathrm{MH})$ S. aureus usando un hisopo de algodón. Los discos de NPs y de los nano- compuestos con diferentes concentraciones de NPs se depositaron sobre el agar inoculado, con al menos 3 cm de separación una de otra y no más de 5 discos por placa de agar. Cabe mencionar que el ensayo se realizó por triplicado. Las placas con agar se incubaron en un ambiente aeróbico a $37^{\circ} \mathrm{C}$, de 18 a $24 \mathrm{~h}$. Al día siguiente se registraron las zonas de inhibición, de cada sistema en estudio, para cada una de las bacterias analizadas, se realizaron los cálculos respectivos para obtener la media de las tres repeticiones realizadas.

\section{Ensayos de citotoxicidad}

Se utilizó la línea celular HeLa (ATCC CCL-2), fueron crecidas utilizando Dulbecco's Modified Eagle Medium (DMEM) y en el medio RoswellPark Memorial Institute (RPMI-1640), respectivamente, fueron incubadas a 37 ${ }^{\circ} \mathrm{C}$ en una cámara de $\mathrm{CO}_{2}$ al $5 \%$. Buffer fosfatos, suero bovino fetal, glutamina y piruvato de sodio (Gibco), y bromuro de 3-(4,5-dimetiltiazol-2-ilo)-2,5-difeniltetrazol (MTT, Sigma Aldrich).

Las células fueron cultivadas en placas de 96 pocillos, donde se sembraron 8,000 células por pozo y se incubaron con los adhesivos en forma de látex sin y con NPZnO, para determinar la citotoxicidad. Después de 24 h, se midió la viabilidad celular mediante el ensayo MTT ${ }^{[8]}$. Para ello, se añadió a cada pocillo $80 \mu \mathrm{L}$ del reactivo MTT a una concentración de $5 \mathrm{mg} / \mathrm{mL}$ (SigmaAldrich St. Louis, Mo. USA), y se incubaron por 4 h.

\section{Lectura de resultado}

Pasadas las $4 \mathrm{~h}$ se retiró el sobrenadante de cada pocillo y se añadieron $800 \mu \mathrm{L}$ de DMSO (Dimetilsulfóxido) para disolver los cristales formados. $200 \mu \mathrm{L}$ se transfirieron a una caja de 96 pozos para realizar la lectura a $595 \mathrm{~nm}$ en un lector de placas de ELISA marca BioRad. El control sin NPs se tomó como un 100\% de viabilidad celular y a partir de este punto se calculó el porcentaje de viabilidad del resto de los pocillos con la siguiente fórmula. \% de viabilidad = (lectura $595 \mathrm{~nm}$ tratamiento/lectura $595 \mathrm{~nm}$ control sin tratamiento) X100. 


\section{RESULTADOS Y DISCUSIÓN}

El patrón de difracción de XRD de las NPZnO obtenidas a partir del método de precipitación química se muestra en la Figura 1.

Las nanopartículas obtenidas presentaron una estructura hexagonal tipo wurtzita correspondiente al ZnO. El diámetro promedio de partícula se calculó a través de la ecuación de Debye-Scherrer [9], usando el ancho medio máximo $\beta$ de las líneas de difracción de rayos $\mathrm{X}$.

$$
\mathrm{D}=\frac{\mathrm{K} \lambda}{\beta \cos \theta}
$$

Donde, D es el diámetro de la partícula, $\mathrm{k}$ es la constante de scherrer, $\lambda$ es la longitud de onda de los rayos $\mathrm{X}, \beta$ la anchura del pico a la mitad del máximo, $\theta$ es el ángulo de difracción de Bragg. De acuerdo con esta ecuación el diámetro determinado de las NPZnO mediante esta técnica fue de $17 \mathrm{~nm}$ aproximadamente.

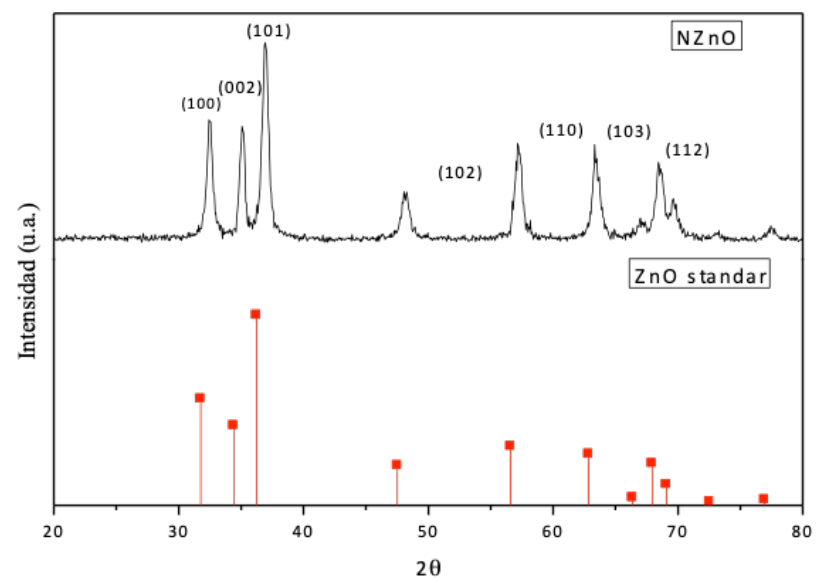

FIGURA 1. Patrón de difracción de Rayos-X de las NPZnO.

La Figura 2a y b muestra una micrografía de TEM de las NPZnO sin modificación y la imagen de las NPs modificadas superficialmente con APTES, respectivamente. La Figura 2a muestra las NPZnO, las cuales presentan un tamaño promedio de $41 \mathrm{~nm}$, estas tienden aglomerarse debido a la alta energía superficial de las nanopartículas. La Figura 2b muestra la imagen de las NPs modificadas en la que se observa un recubrimiento depositado sobre la superficie de las nanopartículas como resultado de la modificación. El espesor del agente de modificación no se aplicó uniformemente sobre la superficie de las NPZnO, como se puede apreciar en la Figura 2b. El espesor del recubrimiento de las nanopartículas se relaciona con el tiempo de funcionalización, temperatura y relación molar utilizada entre otros. El recubrimiento puede estar unido químicamente a la nanopartícula por medio de enlaces Si-O o enlaces covalentes, ya que durante el tratamiento se generan algunos radicales libres del $\mathrm{ZnO}$ en la superficie de las nanopartículas y estos pueden interactuar químicamente ${ }^{[10]}$. Esto produce que las nanopartículas tienden a tener una mejor dispersión cuando son incorporadas a una matriz polimérica, lo cual es atribuido al impedimento estérico entre las nanopartículas que reduce así su tendencia a aglomerarse [11]. Este efecto puede deberse a la interacción que tienen los agentes de modificación con los monómeros, debido a que hay un cambio en el grado de incorporación de las NPs con los agentes de modificación.

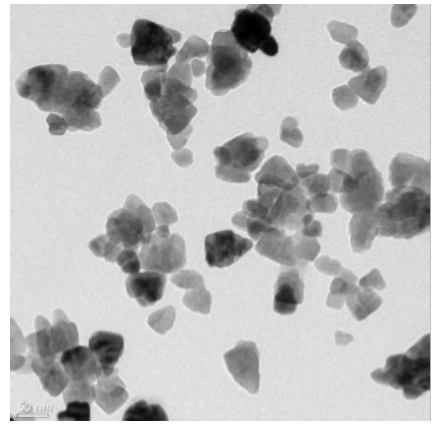

a)

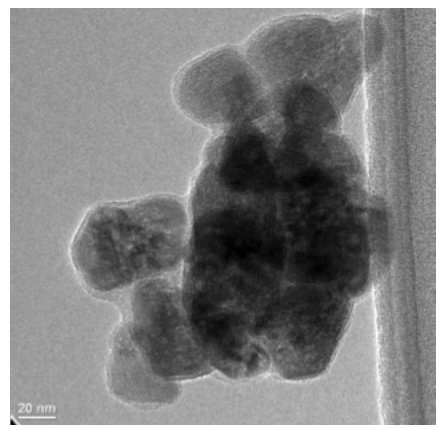

b)
FIGURA 2. Micrografías obtenidas por TEM de las nanopartículas de ZnO sin modificar a) y b) modificadas con APTES.

La modificación superficial de las NPs puede llevarse a cabo en forma individual o como grupos de partículas. Es muy difícil recubrir sólo partículas individuales, ya que es muy conocido que las nanopartículas se adhieren entre sí debido a su alta energía superficial ${ }^{[10]}$. 
En la Figura 3 se muestra el espectro infrarrojo de los PSA con y sin NPZnO al 0.3\%. En ambos casos, las bandas características de IR corresponden al estiramiento del enlace $\mathrm{C}=\mathrm{O}$ de los grupos ésteres presentes en el copolímero en $1734 \mathrm{~cm}^{-1}$. De igual manera las bandas de 1241-1161 $\mathrm{cm}^{-1}$ corresponden a la flexión del enlace $\mathrm{C}-\mathrm{O}$, mientras las bandas de estiramiento de las cadenas saturadas (C-H) aparecen entre $3000-2880 \mathrm{~cm}^{-1}$, las cuales corresponden a los metilos y metilenos presentes ${ }^{[12]}$. A diferencia del espectro infrarrojo del PSA, en el FT-IR del PSA/NPs también aparece una señal de alrededor $1578 \mathrm{~cm}^{-1}$, la cual corresponde a los residuos de monómeros $(C=C){ }^{[13]}$, esto debido a que no se alcanzó una total conversión. Adicionalmente, también en el espectro infrarrojo del PSA/NPs aparece una banda alrededor de $618 \mathrm{~cm}^{-1}$, la cual corresponde a la banda de flexión del enlace $\mathrm{Zn}-\mathrm{O}{ }^{[14]}$.

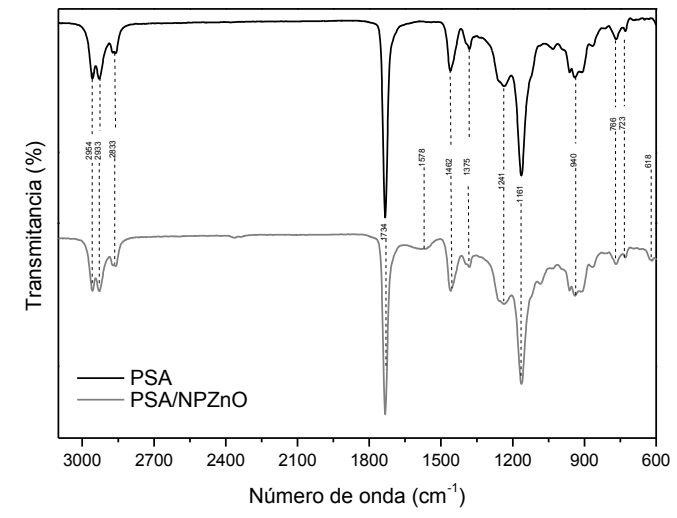

FIGURA 3. Espectro infrarrojo de los adhesivos sensibles a la presión (PSA) antes y después de la adición de nanopartículas.

La Tabla 1 presenta el estudio de las pruebas DSC que fue realizado a los adhesivos PSA/NPZnO, PSA/NPZnODMSO, PSA/NPZnO-APTES utilizando diferentes concentraciones de NPZnO (Tabla 1). En el PSA con menor concentración de nanopartículas sin modificar muestra una Tg de $-59.35^{\circ} \mathrm{C}$ la cual se incrementa conforme se incrementa la concentración de nanopartículas a $-47.9^{\circ} \mathrm{C}$, mientras que con las NPZnO-APTES y NPZnODMSO se observa también el mismo efecto debido a que la estructura de los adhesivos se vuelve más rígida en presencia de las nanopartículas. Este comportamiento produce un incremento en las propiedades de pegajosidad (Tack) y adhesión de los adhesivos, además que nos indica que las NPZnO producen una alta estabilidad térmica, ya que retrasan la transmisión rápida de calor y lo que puede limitar la descomposición del material ${ }^{[15]}$.

\section{TABLA 1. Transición vítrea (Tg) de nanocompuestos de PSA con diferentes partículas.}

\begin{tabular}{|c|c|c|c|c|c|}
\hline \multirow{2}{*}{ Muestra } & \multicolumn{5}{|c|}{ Contenido de nanopartículas (\%) } \\
\hline & \multirow{5}{*}{$\underbrace{\circlearrowright}_{\substack{\infty \\
\bullet}}$} & 0 & 0.1 & 0.2 & 0.3 \\
\hline PSA & & -59.35 & -- & --- & --- \\
\hline PSA/NPZnO & & --- & -50.29 & -47.9 & -50.71 \\
\hline $\begin{array}{l}\text { PSA/NPZnO- } \\
\text { APTES }\end{array}$ & & --- & -49.21 & -49.51 & -48.63 \\
\hline $\begin{array}{l}\mathrm{PSA} / \mathrm{NPZnO}- \\
\mathrm{DMSO}\end{array}$ & & --- & -48.3 & -69.29 & -51.9 \\
\hline
\end{tabular}

\section{Actividad antimicrobiana}

Una vez modificadas las nanopartículas, se procedió a analizar el efecto antimicrobiano de éstas en comparación con las no modificadas, así como su efecto cuando son incorporadas a los PSA. Estos análisis se realizaron utilizando la técnica de difusión de disco en agar para los microorganismos $S$. aureus y S. pyogenes como se muestra en la Figura 4. Las NPs sin modificar y modificadas superficialmente exhibieron un efecto inhibitorio sobre el crecimiento bacteriano, para cada microorganismo. Este efecto puede ser debido a que como las bacterias Gram positivas carecen de una membrana protectora alrededor de las capas de peptidoglicano en la pared celular, esto les permite interactuar y romper la membrana externa más fácilmente y así inhibir, con mayor eficiencia, el crecimiento de bacterias Gram positivas ${ }^{[16]}$. La diferencia en la actividad antimicrobiana de las NPs modificadas y no modificadas se relaciona con el número de vacancias de oxígeno que son típicas de las propiedades del ZnO. Un aumento en el número de vacancias de oxígeno hace que las NPs se carguen positivamente 
y, por lo tanto, realza las interacciones electrostáticas entre las NPs. Para el caso de las NPs modificadas, el efecto inhibitorio se puede atribuir a la presencia de los enlaces Si-O que dan una buena cobertura superficial a las NPs y actúan como una barrera energética. La modificación superficial genera un aumento en la permeabilidad de la membrana y la penetración celular. Esto puede dar lugar a una disminución en la cantidad de proteína en las células expuestas en las NPs modificadas porque el $\mathrm{ZnO}$ es altamente reactivo con estas moléculas ${ }^{[17]}$.

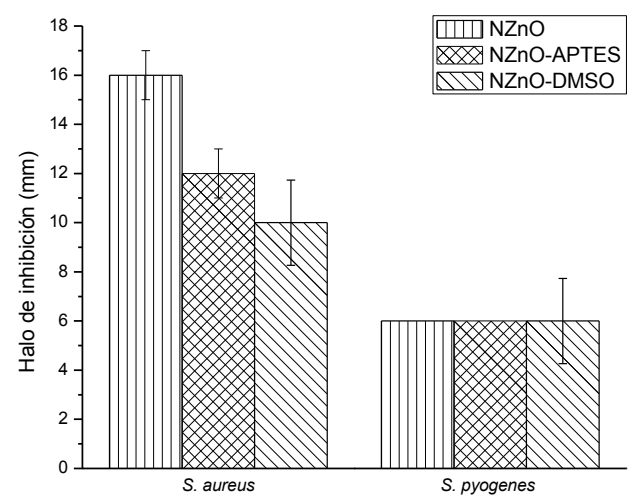

FIGURA 4. Halos de inhibición de las

nanopartículas de óxido de zinc sin y con modificación frente a S. aureus y S. pyogenes.

La actividad antimicrobiana de los PSA con las NPs modificadas y sin modificar presenta un efecto inhibitorio muy similar contra ambos microorganismos cuando se incrementa la concentración de NPs en los PSA, ya que generan un aumento en el número de moléculas de oxígeno activo que causan la muerte celular. Lo que sugiere que las NPs se adhieran a la membrana celular interrumpiendo su respiración e interactuando con un tipo particular de enzimas causando así la muerte celular. Los resultados obtenidos nos indican que el uso de NPZnO sin modificar y modificadas como agentes antimicrobianos en matrices poliméricas produce grandes beneficios para la salud ya que estos microorganismos no generan resistencia a este tipo de NPs, contrario a la resistencia que pueden generar a algunos antibióticos (Figura 5).

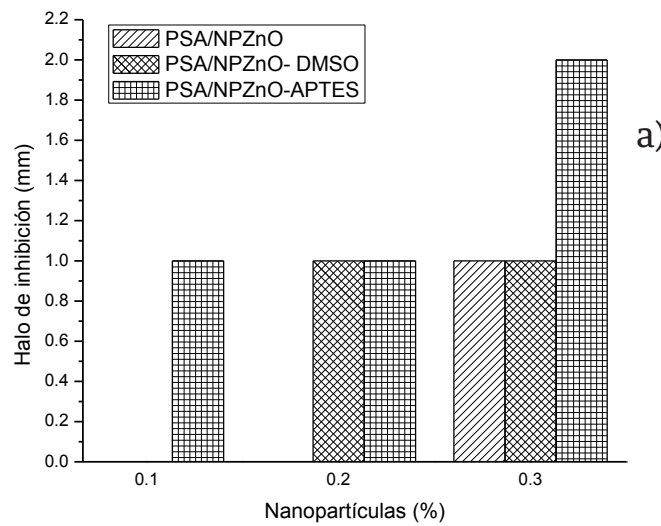

a)

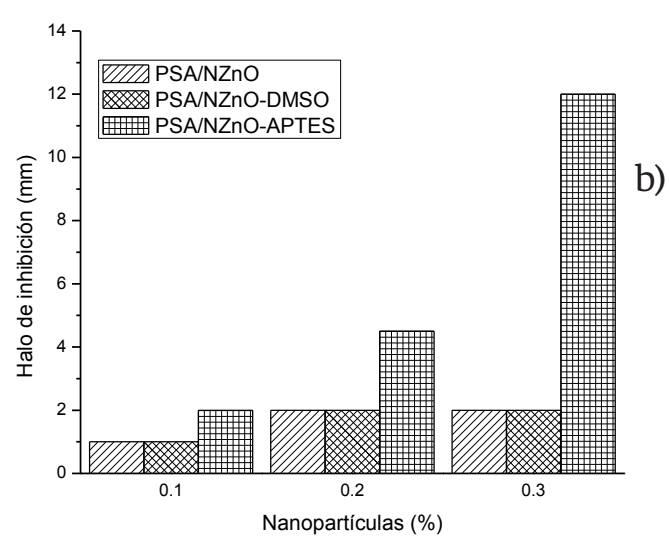

FIGURA 5. Halos de inhibición de los nanocompuestos frente a a) S. aureus y b) S. Pyogenes.

\section{Viabilidad celular}

Los resultados de viabilidad celular evaluada mediante el método MTT, señalan que los PSA sin y con la presencia de NPZnO (Figura 6), poseen una viabilidad del 90 y $80 \%$ con respecto al control sin tratamiento. Esta disminución en la viabilidad celular puede ser atribuida a la liberación del ión $\mathrm{Zn}^{+2}$, el cual es capaz de penetrar en compartimiento ácido de los liposomas e incrementar la generación de especies reactivas de oxígeno (ROS) ${ }^{[18]}$ Se sabe también que el incremento en la disolución de los iones $\mathrm{Zn}^{+2}$ en el medio, es capaz de incrementar la citotoxicidad de las partículas, por lo cual es posible que los iones metálicos liberados por NPZnO contribuyeran a la toxicidad [19]. Sin embargo NPZnO, en las concentraciones añadidas, no resultan ser tóxicas, para las células HeLa acorde a la norma. 


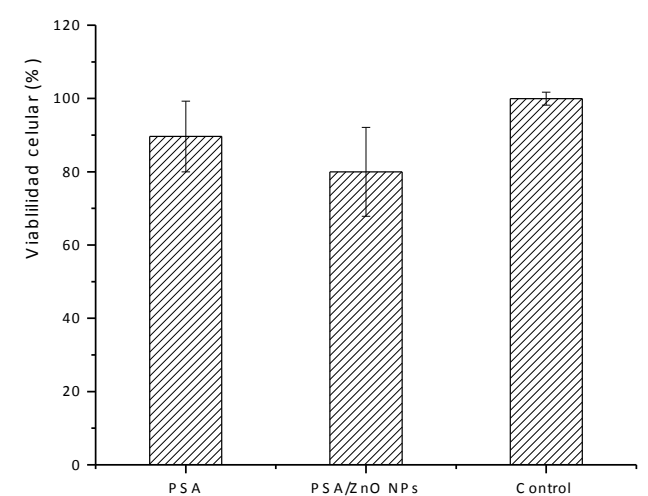

FIGURA 6. Viabilidad celular de los adhesivos sensibles a la presión (PSA) con y sin de nanopartículas.

\section{Resistencia al pelado (Prueba de adhesión a 180)}

Las propiedades de resistencia a la adhesión de los PSA preparados son las que definen el desempeño y uso final de los PSA (Figura 7). Los PSA sin y con la presencia de NPs muestran un marcado efecto en las pruebas de resistencia al delaminado (peel sterngth). En la muestra que contiene la mayor concentración de NPs sin modificar muestra un valor de $1.1 \mathrm{~N} / 25 \mathrm{~mm}$ que es el que presenta mejores propiedades. Esto se puede relacionar con el comportamiento en la $\mathrm{Tg}$ puesto que el incremento en la Tg produce un incremento en la adhesión ${ }^{[20]}$. Siendo estos resultados bastante adecuados para pegarse y despegarse de manera segura a la piel y alrededor de la herida, lo que ocasionaría menor trauma al ser removido de la piel.

\section{CONCLUSIONES}

Se sintetizó un copolímero de 2-EHA/MMA al cual, después de añadirle NPZnO presenta diferencias en composición y por consecuencia en su Tg. La modificación de las nanopartículas de $\mathrm{ZnO}$ incrementa las

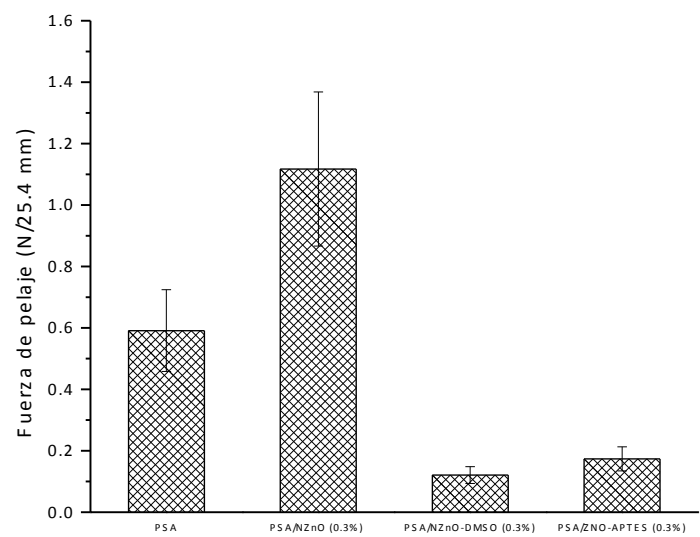

FIGURA 7. Fuerza de pelado de los nanocompuestos con diferentes nanopartículas.

propiedades antimicrobianas frente a $S$. aureus y $S$. pyogenes, obteniéndose actividad antimicrobiana desde $0.1 \%$ del PSA-NPs. La adhesión se ve alterada por la modificación de las nanopartículas presentándose una mayor adhesión en el PSA-NPZnO. Esto es debido a que la modificación de las nanopartículas disminuye la presencia de grupos $\mathrm{OH}$ superficiales los cuales son responsables de la adhesión. En cuanto a la citotoxicidad, se muestra que ésta disminuye cuando se le incorporan NPs, sin embargo, esta disminución no es significativa.

\section{AGRADECIMIENTOS}

El primer autor agradece el apoyo al Consejo Nacional de Ciencia y Tecnología (CONACYT) por la

beca 263277 otorgada por cursar el doctorado de Tecnología en Polímeros del Centro de Investigación en Química Aplicada. Los autores agraden al CONACYT a través del Laboratorio Nacional de Grafeno (CONACYT-232753) por las facilidades otorgadas. También se agradece el apoyo brindado a: A. Espinoza, F. Zendejo, R. Cedillo, S. Zertuche, A. Herrera. 


\section{REFERENCIAS}

[1] Rasmussen JW., Martínez E, Louka P, Wingett DG. (2010). Zinc oxide nanoparticles for selective destruction of tumor cells and potential for drug delivery application. Expert Opin Drug Deliv. 7(9), 1063-1077. doi.10.1517/17425247.2010.502560

[2] Mascorro R., Dorantes H. Y Corea M. (2012). Morphology influence over the peeling property in pressure-sensitive adhesives. Rev. Mex. Ing. Quim., 11(2), 323-331.

[3] Jingyeong O. (2009). Synthesis and Adhesion Performance of Polyacrylate-clay Pressure-sensitive Adhesive as nanocomposite by in-situ polymerization. Tesis de Maestría en Ciencias Forestales. Universidad Nacional de Seúl, Corea.

[4] Lofton L. (2004). Clay/polymer nanocomposites for pressure sensitive adhesives. Rohm and Haas Company, Spring House, PA.

[5] Kajtna, J., Šebenik U. (2009). Microsphere pressure sensitive adhesives-acrylic polymer/montmorillonite clay nanocomposite materials. International Journal of Adhesion and Adhesives 29(5), 543550. doi.10.1016/j.ijadhadh.2009.01.001

[6] Rana, P.K. Sahoo P.K. (2007). Synthesis and pressure sensitive adhesive performance of Poly (EHA-co-AA)/Silicate nanocomposite used in Transdermal drug delivery. Journal of Applied Polymer Science 106(6), 3915-3921. doi.10.1002/app.27034

[7] Wang, T., Lei C.-H, Dalton A. B., Creton C., Lin Y., Fernando K.A.S., Sun Y.P., Manea M., Asua J.M., Keddie J.L. (2006). Waterborne, nanocomposite pressure-sensitive adhesives with high tack energy, optical transparency, and electrical conductivity. Advanced Materials,. 18(20), 2730-2734.

doi.10.1002/adma.200601335

[8] Mosmann T. AshaRani PV, Low Kah Mun G, Hande MP, Valiyaveettil S. (1983). Rapid colorimetric assay for cellular growth and survival: Application to proliferation and cytotoxicity assays. J Immunol Methods 65(1-2), 55-63. doi.10.1016/0022-1759(83)90303-4

[9] Chen, L., Zhengb L., Lva Y., Liua H., Wanga G., Rena N., Liua D, Wanga J., Boughtonc R.. Surface and Coatings Technology, 2010. 204(23): p. 3871-3875.

[10] Esteves A.C., Timmons A.B., Trinidae T. Nanocompositos de matriz polimérica: estratégias de síntese de materiais híbridos. (2004) Quim. Nova. 27 (5) 798-806.
[11] Tang E., Cheng G., Ma X., Pang X., Zhao Q. (2006). Surface modification of zinc oxide nanoparticle by PMAA and its dispersion in aqueous system. Applied Surface Science 252(14), 5227-5232. doi.10.1016/j.apsisc.2005.08.004

[12] Kagel N. (1971) Infrared Spectra of Inorganic Compounds Chemical Physics Research Laboratory. Academic Press, Inc.

[13] Taghizadeh SM, Ghasemi D. (2010). Synthesis and Optimization of a Four-component Acrylic-based Copolymer as Pressure Sensitive Adhesive. Iranian Polymer Journal. 19(5), 343-352.

[14] Purcar V., Somoghi R., Georgiana M., Nicolae C., Alexandrescu E., Gîfu I., Gabor A., Stroescu H., Ianchis R., Căprărescu S., Cinteză L. The Effect of Different Coupling Agents on Nano-ZnO Materials Obtained via the Sol-Gel Process. Nanomaterials. 2017. 7, 493.

[15] Jovanović, R., Dubé M.A. (2004). Emulsion-Based PressureSensitive Adhesives: A Review Journal of Macromolecular Science, Part C 44(1), 1-51. doi.10.1081/MC-120027933

[16] Emami-Karvani Z., C. P, African Journal of Microbiology Research, 2011. 5(12): p. 1368-1373.

[17] Yousef J. and Danial. N.E., Journal of Health Sciences, 2012. 2(4): p. 38-42.

[18] Vasile O., Serdarua I., Andronescua E., Truşcă R., Surdua V., Oprea O., Iliea A., Vasile S. (2015). Influence of the size and the morphology of ZnO nanoparticles on cell viability. Comptes Rendus Chimie 18(12), 1335-1343. doi.10.1016/i.crci.2015.08.005

[19] Zhang X.,Wang Z, Mao L., Dong X., Peng O., Chen J., Tan C., Hu R. (2017). Effect of ZnO nanoparticle on cell viability, zinc uptake efficiency, and zinc transporters gene expression: a comparison with $\mathrm{ZnO}$ and $\mathrm{ZnSO} 4$. Czech J. Anim. Sci. 62(1), 32-41. doi.10.17221/15/2016-CJAS

[20] Wu, L., Wang M., Zhang X., Chen D., Zhong A. (2009). Organic montmorillonite modified polyacrylate nanocomposite by emulsion polymerization. Iranian Polymer Journal 18(9), 703-712. 